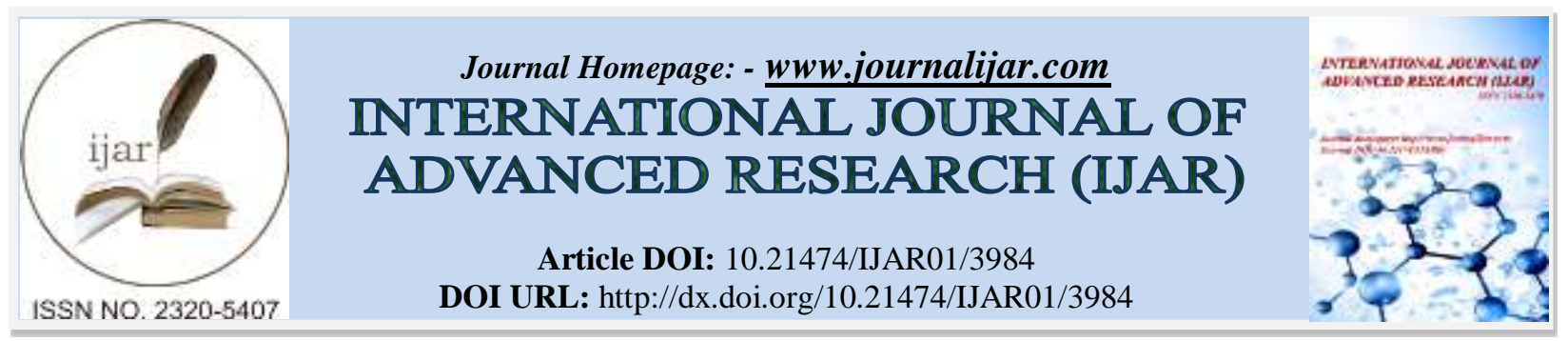

RESEARCH ARTICLE

\title{
SOCIAL MEDIA MESSENGER: USEFUL TOOL FOR DECISION MAKING IN OBSTETRIC EMERGENCY DUTY HOURS.
}

\section{Dr. Shailesh Jain, Dr. Meeta Gupta and Dr. Rakesh Khuteta.}

\section{Manuscript Info}

Manuscript History

Received: 01 February 2017

Final Accepted: 01 March 2017

Published: April 2017

Key words:-

social media messenger, emergency duty hours.

\section{Abstract}

Aim \& Objective: To evaluate social media messenger usage for communication between consulting and emergency doctors on duty in emergency room of Obstetrics \& Gynecology department (OBGY).To compare social media messenger as communication tool with traditional methods of communication in emergency duty hours.

Method: A retrospective, observational study was conducted in the emergency of OBGY department of our tertiary care hospital between January 2016 and December 2016. A total of 246 consultations were included in the study. The messages that were transferred to consultant gynecologists consisted of $92(37.4 \%)$ photographic images, $240(97.5 \%)$ text messages, $12(4.8 \%)$ videos, and 10 $(4.1 \%)$ voice messages.

Results: Treatment was started within $15 \mathrm{~min}$ in $178(72.3 \%)$ cases and within $30 \mathrm{~min}$ in 56(22.7\%) women. $136(55.3 \%)$ women were shifted within 20 minutes for surgery after the response on messenger. 83(33.7\%) women were shifted to HDU(High dependency unit) and $21(8.5 \%)$ women to ICU after consultation. 13 (5.3\%)critical women were saved due to timely intervention. Most of the consulting physicians were outside of the hospital or were mobile at the time of the consultation $(n=192,78.0 \%)$. The outside consultation request rate was significantly higher for night shifts than for day shifts $(p=.004)$, and the majority of outside consultation request were concluded by only response on application $(p<.001) .44(17.8 \%)$ cases required visit of consulting gynecologist and the gynecologist was available within an average time of 26 minutes.

Conclusion: Social media messenger is useful innovation in emergency healthcare, especially for doctor on duty and who are outside the hospital, because of the ability to transfer large amounts of clinical and radiological data during a short period of time.

Copy Right, IJAR, 2017,. All rights reserved.

\section{Introduction:-}

Social messaging or social messaging applications or chat applications are instant messaging or mobile messaging (Short Message Service/SMS) clients that are built around social networking platforms ${ }^{[1]}$. Some examples of popular social messaging include BlackBerry Messenger, Facebook Messenger, Google Hangouts, KakaoTalk, Line, WeChat, and WhatsApp ${ }^{[2]}$. With these social media messengers, one can send images 
and videos as message. Also voice calls are provided by some of these messengers. Recently, these messengers are preferred as route of communication.

WhatsApp Messenger is a proprietary, cross-platform, encrypted instant messaging client for smartphones. ${ }^{[2]}$ It uses the Internet to send text messages, documents, PDF files, GIF images, video, user location and audio messages ${ }^{[8][9]}$ to other users using standard cellular mobile numbers. As of February 2016, WhatsApp had a user base of one billion, ${ }^{[2]}$ making it the most popular messaging application.

Emergency services in Obstetrics and Gynecology department depends on the level of hospital. In India, Primary health center (PHC) provides basic normal vaginal delivery services and referral facilities if required. Community health center (CHC) provides caesarean section operation facilities and blood storage facilities. Tertiary care hospitals provide all monitoring facilities with operational facilities round the clock. In emergencies team work plays vital role. Everywhere a team of doctors (Gynecologist, anesthetist, pediatrician) provide facilities. Decision making is usually role of senior gynecologists and the operations are done by the team present on the floor. Most of the time decisions are clear and can be taken by the senior duty doctor present on floor. But, sometimes one needs opinion regarding some critical conditions so that better treatment can be provided within short time. Previously, senior doctors were called from their residence in emergency hours to visit the patient and give opinion. This took lot of time and delay in starting treatment. Social media messenger is a recent mobile application which has changed the scenario recently.

\section{Material and Methods:-}

Study was conducted in the emergency of OBGY department of our tertiary care hospital between January 2016 and December 2016. Doctors on floor were advised to consult senior obstetricians through social media messenger whenever required. A total of 246 consultations were included in the study. Obstetric emergencies consulted were pre eclampsia, eclampsia, antepartum hemorrhage, post partum hemorrhage, DIC, severe anemia, ectopic pregnancy, uterine perforation, rupture uterus, woman with perineal injury in shock, medical disorders in pregnancy like acute fatty liver of pregnancy, essential hypertension, heart disease, gestational diabetes mellitus, epilepsy. Decisions were taken by senior obstetricians in these 246 cases and treatment was carried out by the team on floor. Time to initiate treatment after the response was noted. Time taken since admission till shifting the patient to HDU/ICU or OT was noted. Senior obstetricians were called for critically ill patients and time to reach the hospital was noted. Results were compared with traditional methods of communication.

\section{Results:-}

A total of 246 consultations were sought for obstetric conditions. The messages that were transferred to consultant gynecologists consisted of $92(37.4 \%)$ photographic images, $240(97.5 \%)$ text messages, $12(4.8 \%)$ videos, and $10(4.1 \%)$ voice messages [Figure 1]. The median response time of the consultant to WhatsApp messages containing preliminary diagnoses, laboratory test results and radiographic images was $3.5 \mathrm{~min}$ (min-max:1-22 min).In 178(72.4\%) women, treatment was initiated within 15 minutes and in 56(22.7\%) women within 30 minutes[fig 2]. 83(33.7\%) women were shifted to HDU and 21(8.5\%) shifted to ICU after the reponse on messenger[fig 3]. $170(69.1 \%)$ women were shifted to OT for surgery. 122(49.5\%) women were shifted for LSCS for obstetric indications. $13(5.3 \%)$ critical women were saved due to timely intervention. Most of the consulting physicians were outside of the hospital or were mobile at the time of the consultation $(n=192,78.0 \%)$. The outside consultation request rate was significantly higher for night shifts than for day shifts $(p=.004)$, and the majority of outside consultation request were concluded by only response on application $(p<.001) .44(17.8 \%)$ cases required visit of consulting gynecologist and the gynecologist was available within an average time of 26 minutes.

\section{Discussion:-}

Obstetric emergencies need quicker response and prompt action to save the life of mother and the baby. Usually, Obstetric departments have well trained gynecologists on floor. Though, it depends on the facilities you are provided with. In India, a PHC (Primary Health Center) has a doctor who may or may not be trained in obstetrics, accompanied with a nurse. At CHC level (Community Health Center), An obstetrician and pediatrician are usually available with OT facilities and blood storage facilities. At district hospital or medical college level, full team with senior and junior obstetricians, anesthetists and pediatricians are available round the clock with blood bank facilities. Our hospital being a tertiary care center has all facilities and thus receive booked as well as unbooked referred patients from peripheral PHCs, CHCs and district hospitals. Obstetricians are available round the clock and most of 
the decisions are taken by the expert available on the floor. Sometimes, they require a second opinion for obstetric emergencies. For these conditions which require quick decisions, previously protocol was to call the senior obstetrician and then after his advice treatment was started. Now a days, social media messenger has gained popularity in decreasing the response time. Response time to initiate the treatment and /or to shift the patient to HDU/ICU has decreased. In facilities, where HDU/ICU are not available, quick referral to higher center has saved many lives.

To the best of our knowledge, there is no study in obstetrics till date where social media messenger has been used to speed up the diagnosis and tretment process. Gulacti $\mathrm{U}$ et al in 2014 used whatsapp in cases of myocardial infarction to speed up the procedure ${ }^{[3] .}$

Gulacti U et al in 2014 showed role of whatsapp usage in emergency duty hours. There were a total of 614 consultations requested by using the WhatsApp application were evaluated, and 519 eligible consultations were included in the study. The WhatsApp messages that were transferred to consultant physicians consisted of 510 (98.3 $\%)$ photographic images, 517 (99.6\%) text messages, $59(11.3 \%)$ videos, and $10(1.9 \%)$ voice messages. In our study, the messages that were transferred to consultant gynecologists consisted of $92(37.4 \%)$ photographic images, $240(97.5 \%)$ text messages, $12(4.8 \%)$ videos, and $10(4.1 \%)$ voice messages.

The median arrival time of data to consultant was $1.2 \mathrm{~min}$ (min-max: $1-4.5 \mathrm{~min}$ ) whereas in study by Gulacti $\mathrm{U}$ et al it was $3.94 \mathrm{~min}(\min -\max : 1-34 \mathrm{~min}$ ). The median response time of the consultant to WhatsApp messages containing preliminary diagnoses,laboratory test results and radiographic images was $3.5 \mathrm{~min}(\min -\max : 1-9.5 \mathrm{~min})$ whereas it was $2.83 \mathrm{~min}(\min -\max : 1-29 \mathrm{~min}$ ) in study by Gulacti $U$ et al.. Regarding consultation termination, there was a statistically significant difference according to the frequency distribution $(\chi 2=15.36, p=<.001)$ in both the studies. Gulacti U et al showed that in $311(59.9 \%)$ cases, consultation was terminated only by WhatsApp messages; in 207 $(40.1 \%)$ of the cases, consultation was terminated after consulting physicians arrived at the ED whereas in our study, in 202 cases consultation was terminated only by WhatsApp messages; 44 cases required visit by on call gynecologist.

Most of the consulting physicians were outside of the hospital or were mobile at the time of the consultation in both the studies.In our study it was seen in 192(78\%) cases whereas this was seen in 292(56.3\%) consultations in Gulacti u et al study.The majority of outside consultation request were concluded by only response on application $(p<.001)$ in both the studies. The consultation request rate was significantly higher for night shifts than for day shifts ( $\mathrm{p}=.004)$ in study done by Gulacti $\mathrm{U}$ et al which is similar to our study.

Earlier, tradional methods of communication were used. Telephone was the first medium for consultation.An ambulance is used by most of the hospitals to bring the consultant on call and to take decision. Gordon D. Schiff et al analysed delay in 583 cases and most of the delays in starting treatment were due to delay in making diagnosis ${ }^{[4]}$.In a study done by Brender et al on childhood appendicitis, mean professional delay was significantly longer in the group with perforated appendicitis than in the group having appendicitis without perforation $(P<$ $.01)^{[5]}$.Mohsen Taghaddosi et al observed delays in myocardial infarction cases mainly due to delay in diagnosis ${ }^{[6]}$.In our study response time is drastically reduced and definite treatment started within minutes.

\section{Limitations:-}

Study has some limitations.First limitation was availability of wireless internet connection everytime at both ends which resulted in increased time taken to respond. Sometimes doctors on call did not see the whatsapp message immediately on their phone as it was on silent mode and took time to respond.Secondly, as the data was analysed retrospectively, exact time to reach the emergency by on call gynecologist could not be evaluated. So, it was decided to note the time as per instructions given on the medical records.

\section{Conclusion:-}

Social media messenger is useful innovation in emergency healthcare, especially for doctor on duty and who are outside the hospital, because of the ability to transfer large amounts of clinical and radiological data during a short period of time. In present scenario, where most of the governments are working hard to reduce maternal morbidity and mortality, use of social media messenger can be a small but effective step in reducing time to reach diagnosis and start treatment so that a woman can be saved. Pregnant women are usually at risk and quick 
decisions can save lives of both mother and baby. Whatsapp messenger is now a days most common tool used for communication with advance features and can be used at every level of health care.

Conflict of Interest: No conflict of interest between authors.

Funding Disclosure: The authors declare that this study has received no financial support.

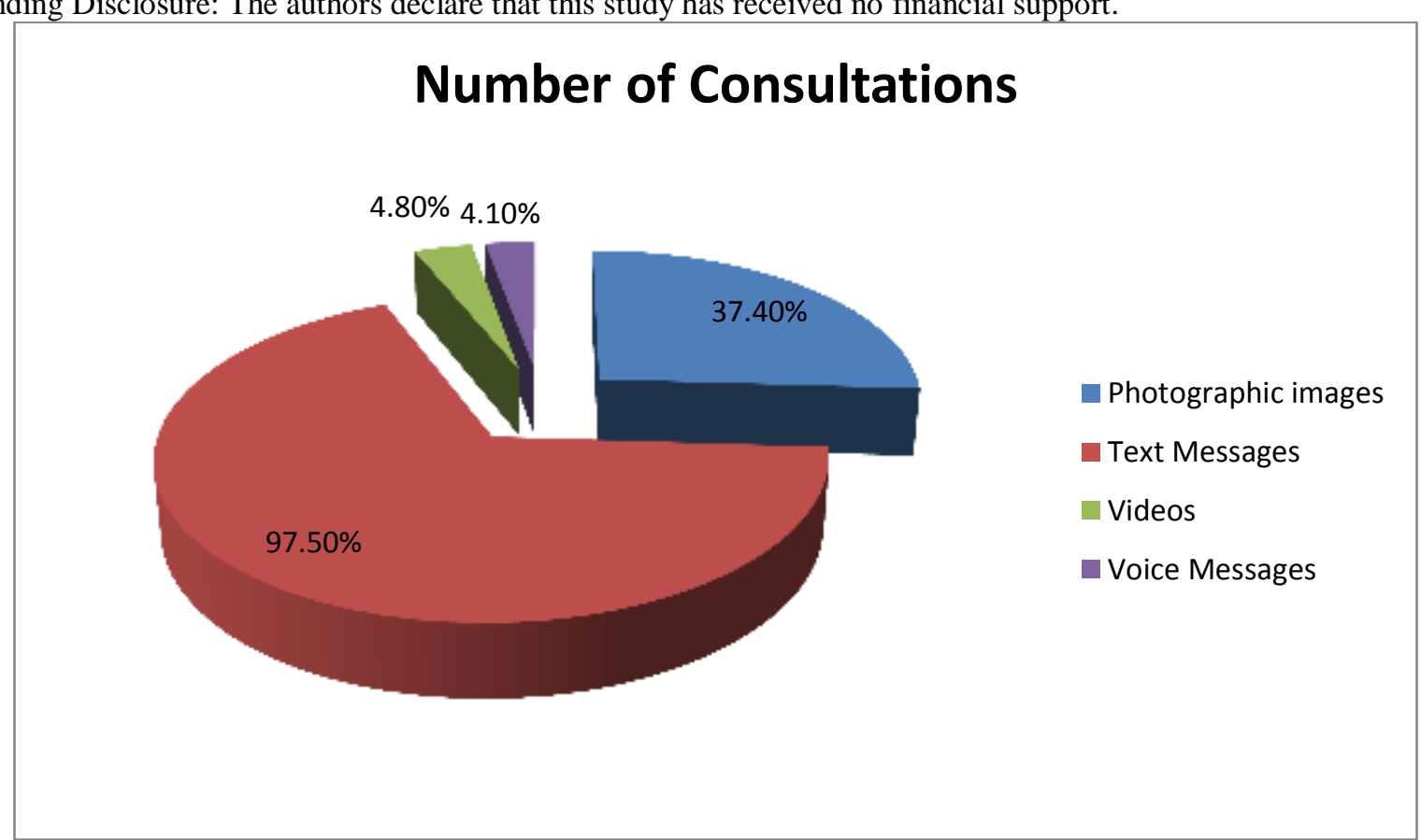

Fig 1:- Showing number of consultations in various forms.

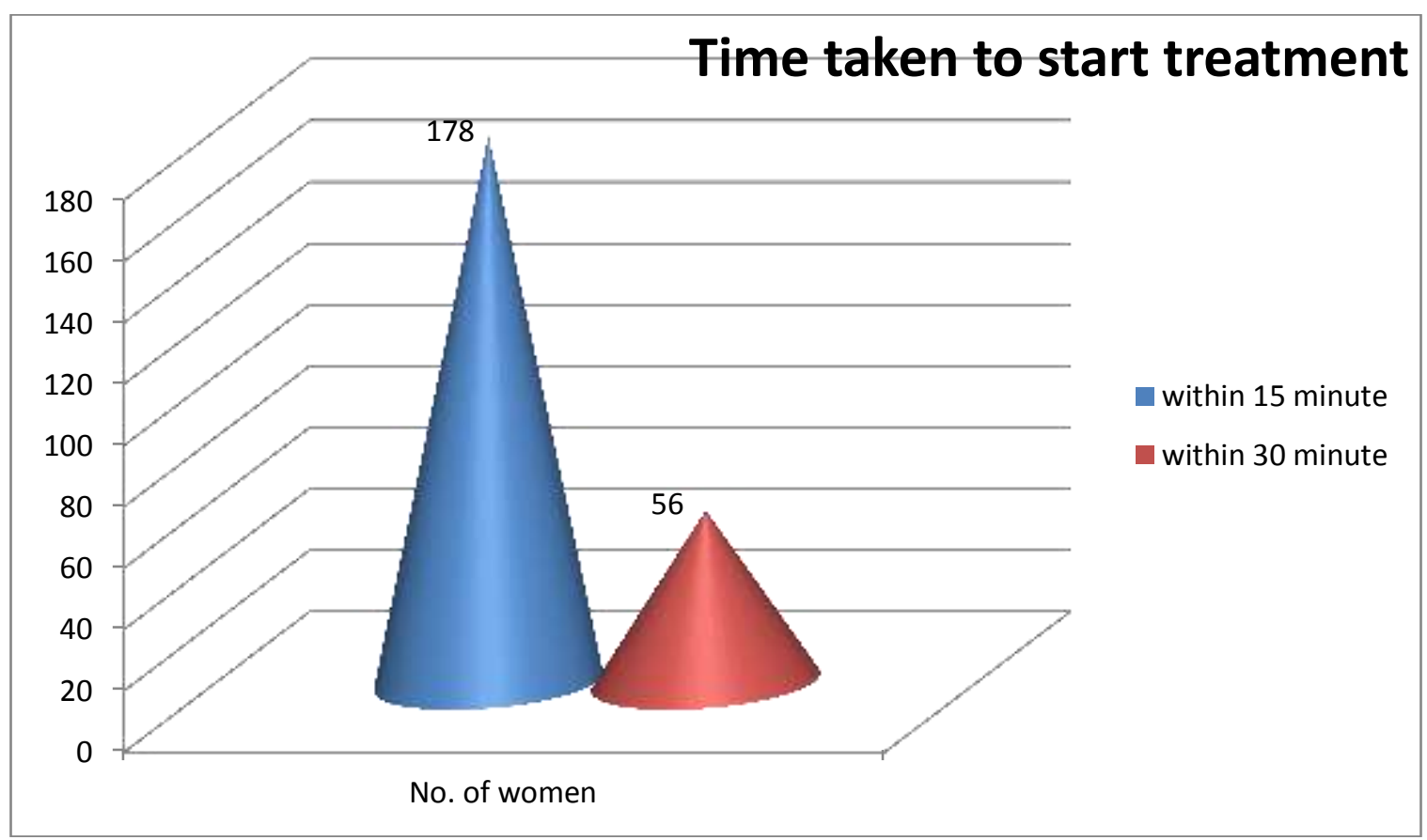

Figure 2:- Time taken to start the treatment after response on social media messenger 


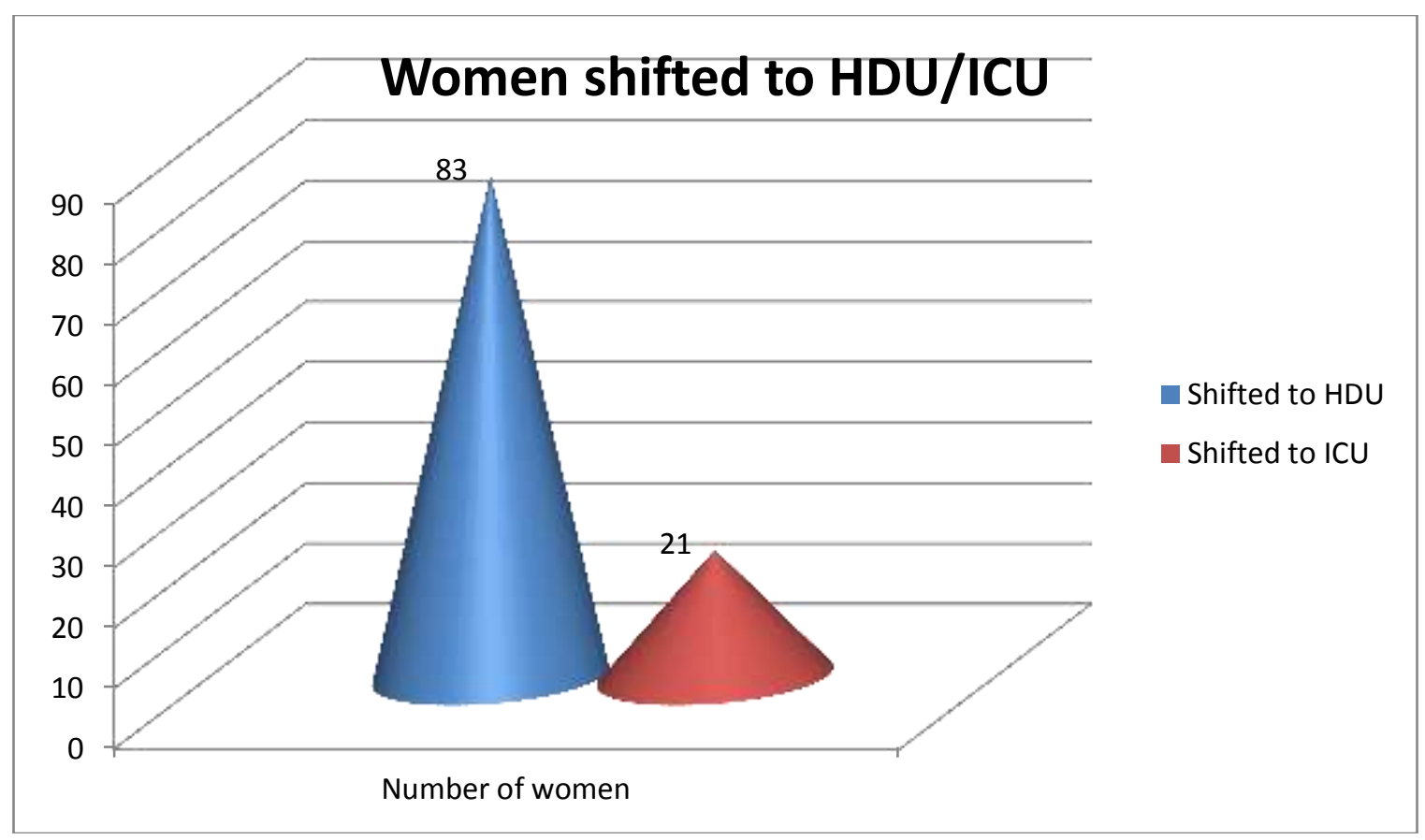

Figure 3:- Number of women shifted to ICU/HDU

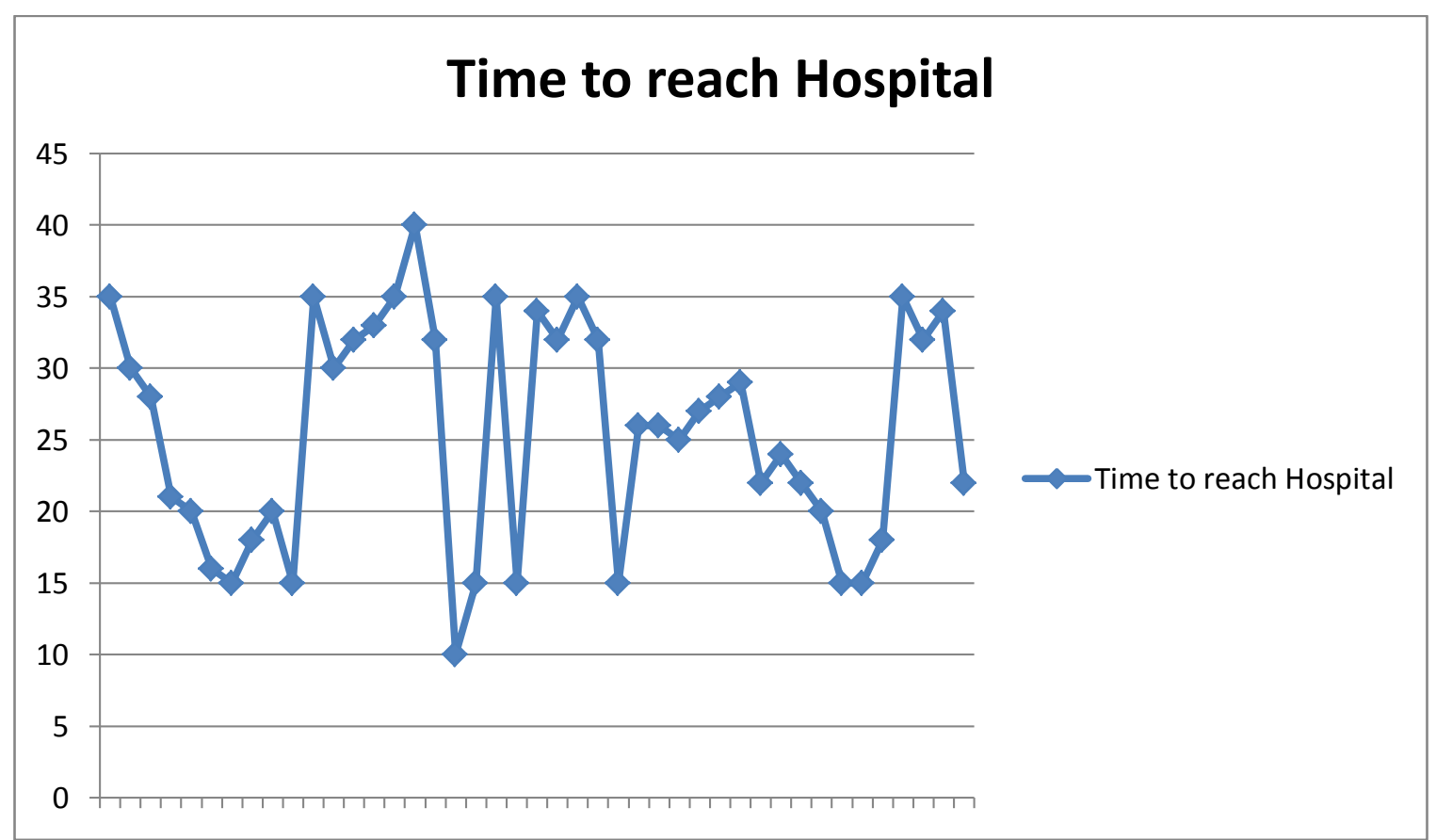

Figure 4:- Time taken by various consultants to reach the hospital for critically ill patients

\begin{tabular}{|l|l|l|l|}
\hline Obstetric Conditions & HDU & ICU & OT \\
\hline Pre eclampsia & 21 & 0 & 9 \\
\hline Eclampsia & 8 & 3 & 2 \\
\hline Postpartum Hemorrhage & 19 & 4 & 5 \\
\hline Antepartum Hemorrhage & - & - & 11 \\
\hline DIC & 0 & 2 & - \\
\hline Severe Anemia & 14 & 3 & - \\
\hline Ectopic pregnancy & 8 & 2 & 6 \\
\hline
\end{tabular}




\begin{tabular}{|c|c|c|c|}
\hline Uterine perforation & - & 2 & 2 \\
\hline Rupture uterus & - & 1 & 1 \\
\hline Perineal injury with shock & 5 & 1 & 6 \\
\hline $\begin{array}{l}\text { Medical disorders } \\
\text { (Acute fatty liver, } \\
\text { Essential Hypertension, } \\
\text { Heart disease, } \\
\text { GDM, } \\
\text { Epilepsy) }\end{array}$ & 10 & 3 & 6 \\
\hline $\begin{array}{l}\text { Obstetric indications for } \\
\text { LSCS }\end{array}$ & - & - & 122 \\
\hline Total & 83 & 21 & 170 \\
\hline
\end{tabular}

Table 1:- Number of women shifted to HDU/ICU/OT after consultation

\section{References:-}

1. WhatsApp Inc. (27 March 2017). "WhatsApp Messenger". App Store. Apple.

2. Metz, Cade (5 April 2016). "Forget Apple vs. the FBI: WhatsApp Just Switched on Encryption for a Billion People". Wired (magazine). Condé Nast.

3. Umut Gulacti, Ugur Lok, Sinan Hatipoglu, Haci Polat. An Analysis of WhatsApp Usage for Communication Between Consulting and Emergency Physicians. J Med Syst (2016) 40:130.

4. Gordon D. Schiff, Omar Hasan, Seijeoung Kim, Richard Abrams, Karen Cosby.Diagnostic Error in Medicine, analysis of 583 Physician-Reported Errors. ARCH INTERN MED/VOL 169 (NO. 20), NOV 9, 2009:1881-1887.

5. Brender, J. D., E. K. Marcuse, T. D. Koepsell, and E. I. Hatch. 1985. "Childhood Appendicitis: Factors Associated with Perforation." Pediatrics 76 (2): 301-06.

6. M. Taghaddosi, M. Dianati, J. Fath Gharib Bigly, J. Bahonaran.Delay And Its Related Factors In Seeking Treatment In Patients With Acute Myocardial Infarction. Arya Atherosclerosis Journal 2010 (Spring); Volume 6, Issue 1,page 35-41. 